A paper to be presented at the 28th Annual Convention of the A merican Institute of Electrical Engineers, Chicago, Ill., June 26-30, 1911.

Copyright 1911. By A. I. E. E.

(Subject to final revision for the Transartions.)

\title{
THE COMMERCIAL LOADING OF TELEPHONE CIRCUITS IN THE BELL SYSTEM
}

\author{
BY BANCROFT GHERARDI
}

The year 1900 may be considered to have marked the beginning of a very important period in the development of telephony. For some time prior to that date it had been known to those who gave study to the matter that the transmission efficiency of long telephone circuits would be improved by increasing the uniformly distributed inductance of the circuit. This knowledge, however, did not lead to any direct commercial results for the reason that no one was able to point out any practical method of increasing the distributed inductance of a telephone circuit without bringing in difficulties of one kind or another which were fatal.

This apparently insuperable difficulty in obtaining improved results by the use of distributed inductance directed the attention of the mathematicians and physicists to the question of whether the results sought for might not be attained by the use of inductance coils placed at intervals in the circuit instead of by undertaking to distribute the inductance uniformly along the circuit. Vaschy, Heaviside and others either suggested or unsuccessfully tested the insertion of self-induction in coils on actual lines as a means of increasing their transmission efficiency. No practical results followed from their work and no actual progress was made in the matter of using lumped inductance to improve the transmission efficiency of telephone circuits until the year 1900, when the patents of Professor M. I. Pupin, dealing with the reducing of attenuation of electrical waves,

Note:-This paper is to be presented at the 28th Annual Convention of the A. I. E. E., Chicago, June 26-30, 1911. Notice of oral discussion or any written discussion should be mailed to reach the Secretary before the date of the meeting. Written discussion received within 30 days thereafter will be treated as if presented at the meeting. 
were issued. Professor Pupin, ${ }^{1}$ and Dr. George A. Campbell, ${ }^{2}$ who had also worked on this problem, have published several papers dealing with it. These authors discuss fully the mathematical theory of improving the transmission efficiency of telephone circuits by means of placing thereon inductance coils at intervals. The papers referred to establish the fact that inductance in coils spaced suitably along a telephone circuit will produce the same effects as uniformly distributed inductance and show us how to suitably locate these inductance coils. The matter of suitably spacing the inductance coils is of vital importance and must be kept in mind because the spacing of the inductance coils is the key to the use of lumped inductance and it is from the failure to establish this fact and give due weight to it that the earlier workers failed.

I shall not undertake here to go over the theoretical ground which has already been so well covered in the papers referred to, but before describing some of the practical applications of loading to open wire lines and cables, and discussing the results which have been obtained and mentioning some of the difficulties which have been overcome, I will state the results of the fundamental work on this question for those who do rot care to follow through the mathematical papers on this subject.

In the study of any telephone line, with reference to its efficiency as a means of transmitting speech, two important factors must be ${ }^{\prime}$ considered. These we ordinarily characterize as "volume" and "quality."

Volume refers to the loudness of the sound which may be obtained from the telephone receiver at the distant end of the line, and the efficiency of the line as regards this factor is determined by the ratio of the amount of energy received at the distant end of the line to the amount of energy put into the line at the transmitting end.

Quality refers to the clearness of the speech which may be obtained from the current at the receiving end of the line. The efficiency of the line as regards quality is determined by the degree that the shape of the current wave at the receiving end of the line approximates to that of the current wave impressed upon

1. Transactions of American Mathematical Society, page 259, July, 1900; Transactions of A. I. E. E., XVII, May, 1900; Transactions of A. I. E. E., XV., March, 1899; Electrical World and Engineer, October 12, 1901, page 587.

2. Philosophical Magazine, March, 1903. 
the line at the sending end. A line which would transmit all the frequencies concerned in the telephonic current with equal attenuations of all these frequencies and with the same velocity would transmit without distortion, and its quality might be considered to be perfect.

For the present I will confine my attention to the matter of the volume of speech transmitted. The formula for the attenuation of a telephone circuit, excluding the effects of terminal conditions which react on the portions of the line near the ends, is

$$
\alpha=\sqrt{\frac{1}{2} \sqrt{\left(R^{2}+p^{2} L^{2}\right)\left(S^{2}+p^{2} C^{2}\right)}+\frac{1}{2}\left(S R-p^{2} L C\right)}
$$

in which the symbols have the following significance:

$$
\begin{array}{llll}
\alpha & =\text { attenuation constant } & \text { per unit length. } \\
R=\text { resistance } & \text { " } & \text { " } & \text { " } \\
L=\text { inductance } & \text { " } & \text { " } & \text { " } \\
C=\text { capacity } & \text { " } & \text { " } & \text { " } \\
S=\text { conductance } & \text { " } & & \\
p=2 \pi \text { (frequency). } & & &
\end{array}
$$

This attenuation constant is a measure of the efficiency of the line, for on any line if the amount of current impressed on it at the sending end is known, and the attenuation constant is known the amount of current at any point along the line may be determined by the formula

$$
i=I e^{-m \alpha}
$$

in which $i=$ the current at any point;

$I=$ the initial current, and

$m=$ the distance from the initial point of the line to the point at which the current $(i)$ is to be ascertained. Equation (1) may be simplified somewhat by making

$$
S=0,
$$

as this item $S$ representing leakage and dielectric dissipation, is always made as small as is commercially practicable and is, in most cases, sufficiently small to be negligible in its effect upon the general result obtained. With this simplification, equation (1) becomes

$$
\alpha=\sqrt{\frac{p C}{2}\left(\sqrt{ } R^{2}+p^{2} L^{2}-p L\right)}
$$


An examination of this equation shows that the attenuation constant will be reduced as the inductance of the circuit, $L$, is increased. I have already mentioned the fact that no satisfactory commercial method has been suggested or devised for uniformly increasing the inductance. The result of the work published about 1900 shows us, however, that we can obtain the same favorable effects by placing suitably spaced lumped inductance in the circuit as would be obtained from uniformly distributed inductance without the fatal difficulties encountered in endeavoring to place any considerable amount of distributed inductance. This work shows us that if more than $\pi$ inductance coils per wave length are placed upon a telephone circuit the effect of these coils will be approximately the same as the effect of an equal amount of inductance uniformly distributed along the circuit. If less than $\pi$ coils per wave length are placed the results are unfavorable. As the number of coils per wave length is increased above $\pi$ the effect very rapidly approaches the effect produced by uniformly distributed inductance so that if as many as six coils per wave length are placed on the circuit the effect of the inductance thus placed is within 4 per cent of that of uniformly distributed inductance. The wave length in a telephone circuit is easily computed by the following formula:

$$
\begin{gathered}
2 \pi \\
\sqrt{\frac{1}{2}} \sqrt{ }\left(R^{2}+p^{2} L^{2}\right)\left(S^{2}+p^{2} C^{2}\right)-\frac{1}{2}\left(S R-p^{2} L C\right)
\end{gathered}
$$

in which the symbols have the same meaning as in (1) above. To apply this formula to a circuit which it is proposed to load, it is necessary of course to take account of the reaction of the inductance which is to be added to the circuit in the form of loading coils, because this inductance affects the wave length. Therefore, the inductance value in the formula given above must include the proposed loading. In order that all frequencies which are of importance in connection with the transmission of articulate speech shall be cared for by the proposed loading, the frequency employed in this formula must be the highest frequency which takes a necessary part in the clear transmission of speech. It is not found that it is necessary that this spacing of the loading coils should be absolutely uniform. It is important, however, that the spacing should not depart substantially from uniformity.

Without undertaking here to give the equations dealing with 
the effects of loading on the quality or clearness of speech, it may be stated that it is found both mathematically and experimentally that loading makes the attenuation more nearly equal as between the different frequencies which are important in connection with speech transmission and that loading, therefore, increases the clearness of such speech and improves the quality of the line, particularly on underground cable circuits where, on account of the relatively large amount of capacity in relation to the other characteristics of the circuit, the quality without loading is noticeably inferior on long circuits.

Broadly speaking, it may be stated that what we have done in applying loading to long telephone lines is to adopt the necessary measures to enable us to obtain the advantages of a high potential transmission system, that is, of transmitting the electrical energy from the sending end to the receiving end of the line with a high voltage and a small current as compared with the smaller voltage and higher current which is found on unloaded lines. That this is the result which we are actually accomplishing by loading our lines is shown not only from the study of the equations dealing mathematically with the propagation of telephone currents over loaded and unloaded lines, but it is also further established by actual studies and tests on working lines. We have, therefore, broadly speaking, taken the same step which electric light and power people have taken to improve the efficiency of their long lines. On account, however, of the very different electrical characteristics of telephone lines and currents and high tension electric light and power lines and currents the means which it has been necessary for us to adopt to accomplish this result have been very different from those adopted by the electric light and power engineers.

In the case of power which is to be transmitted at relatively low frequencies, usually 66 cycles a second or less, over lines of relatively short length, say, cables 10 miles long and open wire circuits 100 miles long, it is sufficient in order to transmit the energy with a high voltage and a small current to arrange the receiving end of the line by means of a transformer or otherwise so as to give a great reactance, and to place high potential apparatus at the sending end of the line. Electrically the line is so short that the reactance at the receiving end may be considered to be working directly against the high potential generating apparatus at the sending end and hence the desired result is obtained. With the telephone current, however, which 
is of high frequencies, and telephone lines which are often very long, the situation is entirely different. Increased reactance placed at the receiving end of the line does not make itself substantially felt at all at the sending end of the line. To obtain the proper reactance for high potential sending apparatus to work against, it is recessary, therefore, in the case of telephone lines to distribute the reactance along the line.

The electrical characteristics of open wire lines and of telephone cables are substantially different from each other. The following table gives the constants of two typical telephonc cables and of a typical open wire line:

\begin{tabular}{|c|c|c|c|}
\hline Type of circuit & Cable & Cable & Open wire \\
\hline Loop resistance, ohms per mile............ & 88 & 28 & 10.5 \\
\hline Mutual capacity, microfarads per mile... & 0.07 & 0.07 & 0.008 \\
\hline Inductance, henrys per mile.......... & 0.001 & 0.001 & 0.004 \\
\hline Insulation resistance, megohms per mile........ & $500+$ & $500+$ & 1 to 100 \\
\hline
\end{tabular}

The essential differences between the characteristics of these circuits, from the point of view of loading, will be seen by examining the figures in the above table. In the case of underground cable, the capacity is relatively high and the inductance, on the other hand, is relatively small. On overhead lines, however, the capacity is relatively small and the inductance of the circuit, even before it is loaded, is not negligible. The insulation of cables is constant while that of aerial lines varics with the weather conditions. On account of these differences in the electrical characteristics of these two types of circuit the design of the loading coils used on them, the proper spacing of the loading coils, and the results obtained from loading are simewhat different in the two cases. The principles employed are the same in both cases, but the different characteristics of the lines bring about in the loading different dimensions both electrical and mechanical.

The problem of loading underground cables is as a whole easier than that of loading open wire lines and will, therefore, first be discussed.

\section{Loading; Telephone Cables}

At the present time we are making very general use of two styles of loading for telephone cables, one of which is characterized as "medium " loading and the other " heavy" loading. 
In a few cases a third style of loading, known as " light " loading, is employed. These three styles of loading differ in the amount of inductance placed upon the lines and hence in the improvement in transmission obtained from the loading. The heavy loading, as the term would suggest, places the greatest amount of inductance on the line and requires coils placed at closer intervals. This heavy loading employs about 0.2 henry per mile and the spacing between coils is about $1 \frac{1}{4}$ miles; medium loading provides 0.1 henry per mile with the coil spacing of about $1 \frac{3}{4}$ miles; light loading employs 0.05 henry per mile with a spacing of about $2 \frac{1}{2}$ miles. The coils for these various loadings are made up on similar cores so that the coils differ only in reference to the windings placed thercon. A description of the coil cm-
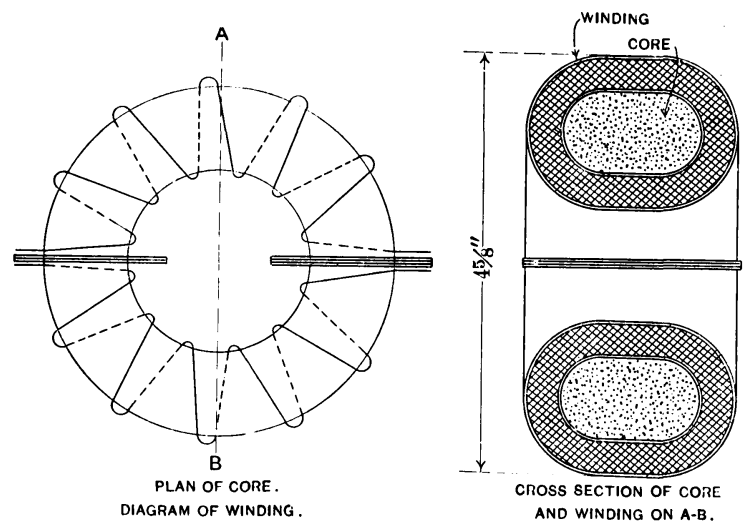

FIG. 1.-Cable loading coil

ployed for medium loading and the method of applying it to the lines is as follows:

The coil is of the toroidal type, that is, it has an iron core like a ring. Fig. 1 shows a cable coil. The core is made up of very fine iron wires. Two copper windings are placed on the core, one to go on each side of the line. These windings are also indicated on Fig. 2 which shows the way the coils are connected in to the line. The windings magnetize the core in the same direction, that is, the mutual induction is added to the selfinduction. Over these windings there is placed a layer of tape for mechanical protection and the whole coil is then thoroughly dried and immersed in a compound to keep out moisture.

Before leaving the design of the coil it is interesting to consider 
the precautions which are taken to reduce losses in it. This is a very important matter as, while the inductance added to the circuit by the coil produces an improvement in the transmission efficiency of the circuit, any loss of energy in the coil means, of course, a reduction in the efficiency of the circuit, and ith poorly designed coils it would be easily possible to bring in losses so large that they would over-balance all the gain from the loading and leave the circuit poorer than before. These losses must be particularly guarded against because they are cumulative.

The first loss to be considered is a dissipation loss resulting from the resistance of the copper winding of the loading coil itself. This loss cannot be entirely eliminated, but it may be reduced as much as may be desired by increasing the cross-section of the winding. There are, however, practical limits to this, as the increase in the size of the winding increases all the mechanical dimensions of the coil and also tends to increase certain other losses.

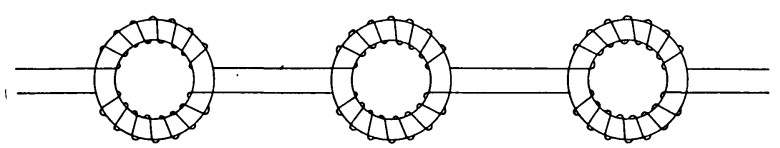

FIG. 2.-Diagram of loading coil windings and method of connecting into a line

A second possible loss in loading coils may also develop in the copper winding. If the cross-section of the conductor used for winding the coil is large, eddy currents may form within the conductor itself and these would produce substantial losses.

Practically this factor is small in cable loading coils, but in open wire coils where a conductor of greater cross-section must be employed it has been found necessary to strand this conductor and to insulate the various strands from each other.

In the iron core of the loading coil there are also several possible losses. In the first place, there is the hysteresis loss. After a great deal of work it has been found practicable to obtain special grades of iron which when workęd within certain limits of magnetization are substantially free from hysteresis losses. This at once imposes a limitation on the design of the loading coil and affects the proportioning of all of its parts.

Another loss which may take place in the core of a loading coil is that due to eddy or Foucault currents which may develop in 
the material of the core itself. In the case of loading coils it has been necessary to construct the cores of extremely fine wire and to coat this wire with a film of insulating material.

Certain other losses must also be guarded against. If a loading coil when magnetized gave an external magnetic field and there were any conducting materials in this fie!d, eddy currents would be produced in this conducting field and would represent a loss of energy through the circuit. Similarly, if there were any iron or other magnetic substances in this field there would be likely to be hysteresis losses in this iron. These losses have been

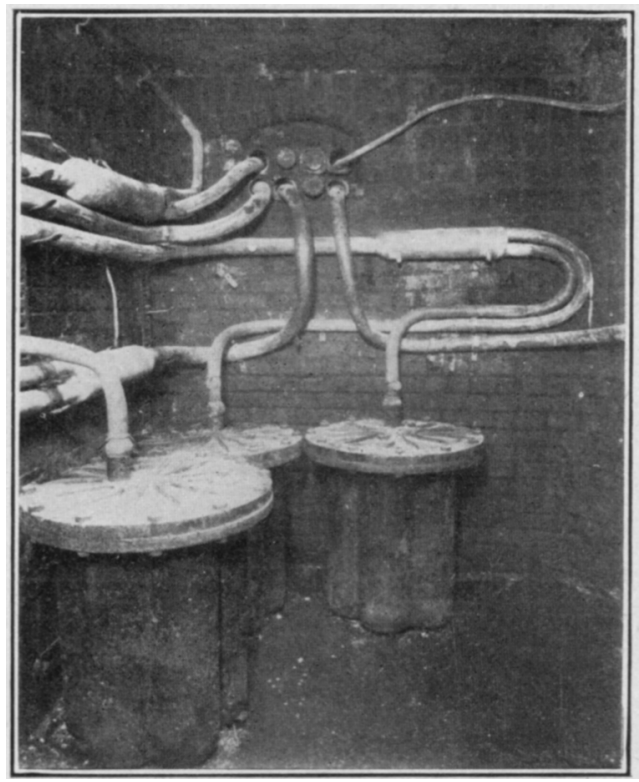

FIG. 3.-Underground man-hole containing loading coils

guarded against by adopting the toroidal form of coil already described and placing the windings on it in such a manner that practically no external magnetic field is in any case produced. The designing of loading coils in this manner has not only eliminated external losses but makes it possible to place loading coils in close proximity to each other without introducing cross talk between the circuits involved. It is interesting to consider that in as far as the losses in loading coils is concerned they very closely resemble the losses which must be guarded against in the construction of, for example, dynamo armatures in electric light and 
power practice. The resemblance, however, stops at this point and the experiences of electric light and power people in connection with these losses have not been of great assistance in the design of loading coils because the magnitude of current, its frequency and other characteristics have been so different from those involved in electric light and power practice. The great importance of reducing losses, which from the nature of loading are cumulative in character, has made the problem very different.

The direct current resistance of the medium loading coil is about four ohms. The alternating current resistance of this coil. at a frequency of about 800 periods a second, is about nine ohms. This relatively small increase of resistance at such a

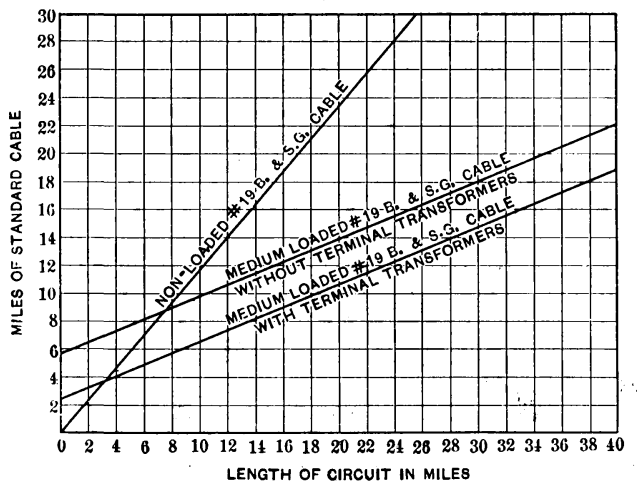

Fig. 4.-Efficiency of No. 19 B. \& S. gauge cable unloaded and medium loaded, with substation apparatus located directly at the ends of the circuits

high frequency is a good indication of the skillful design and careful manufacture of these coils.

The method of placing loading coils in cable circuits is illustrated by Fig. 3, which shows the photograph of a manhole containing loading coil cases. The cases which you see in this photograph each contain 49 loading coils. These are arranged in the case on seven spindles, there being one spindle in the middle of the case and six spindles arranged around it. The case is filled with insulating compound and special precautions are taken between the flanges to exclude water. Extending from the case to the main cable is a stub cable containing the wires leading into and out of the loading coils. This is spliced into the main cable so as to loop a loading coil winding into each side of each circuit which it is desired to load. In some cases where 
it is desired to load aerial cables, cases similar to those shown in Fig. 3 are buried at the base of the pole and the stub containing the wires run up the pole to connect with the aerial cable.

The rest1ts obtained by applying medium loading to No. 19 B. \& S. gauge underground cable, having a capacity of about 0.070 microfarads per mile, are shown in Fig. 4 . In this figure is also shown the transmission efficiency of the unloaded cable. All of these transmission efficiencies are measured in terms of what we call standard No. 19 gauge cable. This standard 19 gauge cable is cable having a resistance of 88 ohms per loop mile and a mutual capacity of 0.054 microfarads per mile. Cables as now manufactured have in general higher capacities than this, but when the earlier transmission tests were made cable having a mutual capacity of 0.054 was in common use and was therefore adopted as the standard and it has been convenient to retain it as such. Wherever in this paper, unless otherwise stated, the efficiency of a circuit is spoken of as being equivalent to so many miles of standard cable or of so many miles of cable, the cable referred to as the unit of measurement is 19 gaugc standard' cable as defined above. It is for this reason that the efficiency of the unloaded cable in Fig. 4 is not quite equal to that of the standard cable used as the unit of measurement.

The efficiencies of the loaded circuits, shown in Fig. 4, are those obtained with substation instruments located directly at the ends of the loaded circuits. One curve is given showing the results obtained by the use of terminal transformers and the other without the use of such devices. These curves bring out clearly a peculiarity of loaded circuits which should be considered at this point. The loading of a circuit, either underground or aerial, brings in at the ends of the loaded circuit whether the circuit is connected directly to substation instruments or to unloaded circuits an effect which we have called reflection losses. That is to say, at the ends of the loaded circuit there are certain losses which take place which are substantially independent of the length of the loaded circuit involved. These losses as between loaded and unloaded circuits are dependent upon the relative electrical characteristics of the loaded and unloaded circuits. In the case of substation instruments, connected directly to the ends of a loaded circuit, the terminal losses are dependent upon the relative electrical characteristics of the substation instrument and of the loaded line. Terminal or reflec- 
tion losses, of course, also exist between unloaded lines of different characteristics, for instance, as between unloaded underground cable and unloaded aerial lines. They would also be found between substation instruments and unloaded lines of various kinds. In all these cases, however, they are small in magnitude and it is only in the case of loaded lines that they assume such proportions that they must be given consideration in connection with general engineering.

I have been speaking of the terminal losses observed in connection with loading as though they pertained particularly to the loaded line. It has been convenient to so speak of them but, of course, they actually pertain to the combination of loaded lines with unloaded lines or standard substation equipment, and properly are no more associated with one than with the other but rather result from a combination of the two. As, however, the substation instrument and the unloaded lines were in service for a considerable time before the use of loaded lines, it has been convenient to associate these reflection losses with the loaded lines and consider the electrical efficiencies of the unloaded lines and the substation instruments to remain unchanged.

Various devices have been successfully employed to reduce terminal losses, although no plan has been worked out which would completely eliminate them. The plan used most successfully to-day for eliminating terminal losses is a device known as a terminal transformer. This is a transformer or repeating coil which differs from the standard telephone repeating coil in that there are more turns on one winding than on the other. It is used in the same way as an ordinary transformer in electric light practice, the high potential side being connected with the loaded line and the low potential side with the unloaded line. For ordinary conditions of loading the ratio of the two windings is usually either 1 to 1.4 or 1 to 2 . The effect of the terminal transformer in reducing terminal losses is well shown in Fig. 4. The terminal loss for the two ends of a medium loaded line without terminal transformers is about, 5.75 miles of standard cable. With terminal transformers this is reduced to 2.5 miles. These figures are for cases where the telephone substation apparatus is located directly at the ends of the loaded line. Where, between the loaded line and the substation apparatus, there is a length of unloaded line it is found that terminal losses are not so great as if the terminal apparatus were directly at the ends of the loaded line. The extent to which the varying amounts of un. 
loaded line reduce terminal losses are shown by the following figures:

\begin{tabular}{|c|c|c|}
\hline \multirow[b]{2}{*}{$\begin{array}{l}\text { Amount of } 19 \text { gauge unloaded cable } \\
\text { at each end of loaded circuit }\end{array}$} & \multicolumn{2}{|c|}{$\begin{array}{l}\text { Amount of terminal losses at both ends } \\
\text { of a loaded circuit-medium loaded }\end{array}$} \\
\hline & $\begin{array}{l}\text { Without terminal } \\
\text { transformers }\end{array}$ & $\begin{array}{l}\text { With terminal } \\
\text { transformers }\end{array}$ \\
\hline None & 5.75 & 2.5 \\
\hline 1 mile & 4.0 & 2.0 \\
\hline 2 miles & 3.0 & 1.50 \\
\hline 3 miles & 2.2 & 1.15 \\
\hline 4 miles & 1.6 & 1.0 \\
\hline 5 miles & 1.25 & 0.9 \\
\hline
\end{tabular}

While terminal losses are substantially independent of the length of the loaded line, they are affected by the character of the loading of the line. The heavier the loading the greater the reflection losses. While with medium loading the reflection losses, as shown by the above table, with no unloaded cable at the ends of the circuit are 5.75 miles without terminal transformers and 2.5 miles with terminal transformers, the corresponding figures for heavy loading are 7.8 miles and 3.5 miles. These figures are, of course, reduced as in the case of medium loading, by having unloaded cable at the ends of the loaded circuit.

If it were not for these terminal losses it would be practicable, theoretically at any rate, to indefinitely increase the efficiency of a 19 gauge circuit by adding more and more inductance to it. Even without terminal losses, however, this could not practically be done, because more and more loading coils would have to be added as more inductance was added, and these would in turn bring in larger and larger losses until finally the further gains from adding inductance would be offset or more than offset by coil losses. The increasing effect of leakage as the weight of loading is increased also has a limiting effect. Aside from these effects, terminal losses, which increase as the amount of inductance added to the circuit is increased, places a limitation beyond which it is not advantageous to increase the amount of loading on the circuits. This is illustrated by Fig. 5, which gives the efficiency of medium and heavily loaded 19 gauge conductors and on which, for the purposes of comparison, the efficiencies of various gauges of unloaded circuits are also given. It will be seen from this that by taking into account the reflection losses 
which are here given at about an average for various conditions, the medium loaded circuit is more efficient than the heavily loaded circuit in lengths up to four miles. Beyond that point the heavily loaded circuit is more efficient, although even at 12 miles the difference is not largely in favor of the heavy loading.

It is interesting to consider from this diagram what results have been obtained from loading 19 gauge conductors. For a length of circuit equal to 4.5 miles the 19 gauge conductor with medium loading is as good as a 16 gauge conductor unloaded; that is to say, loading has made the circuit containing half the copper as good as the larger circuit without loading. For a length of 11 miles a loaded 19 is as good as unloaded 13 weighing four times as much as the 19 gauge conductor.

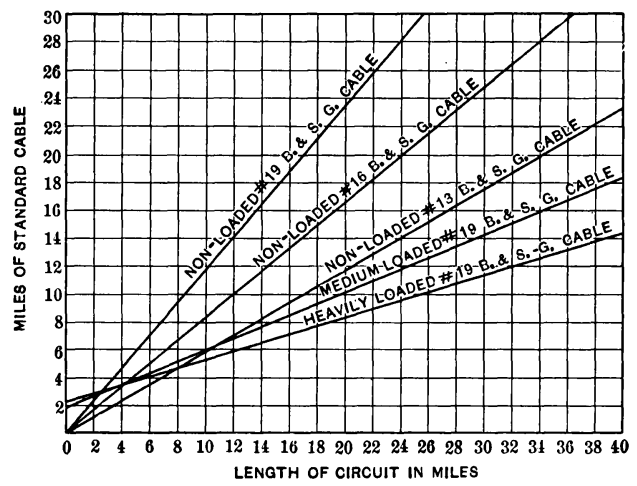

Fig. 5.-Efficiency of No. 19 B. \& S. gauge cable loaded, (medium and heavy), with terminal transformers and one mile unloaded loop at each end; also efficiency of unloaded cables of various gauges

For underground circuits, requiring a greater transmission efficiency than can be obtained by the use of heavily loaded 19 gauge cable circuits, it has been found best, all things considered, to employ a larger gauge of wire rather than to load more heavily. Many cables containing 16, 14 and 13 B. \& S. gauge conductors have been laid and loaded.

The most interesting existing cable containing large gauge conductors is the New York-Philadelphia cable which was laid several years ago between these two points in order to provide additional circuits and to insure the service against storm damage. Most of the circuits in this cable are of $14 \mathrm{~B}$. \& S. gauge and they are heavily loaded. That is, there is 0.2 henry per mile added to the cable circuits by means of loading coils. The results obtained 
on these circuits are given in Fig. 6 assuming average terminal conditions. The results obtained by this loading are most satisfactory. The distance between New York and Philadelphia, following the route of the cable, is 90 miles and this distance is ${ }^{i}$ ndicated on the diagram. It will be seen that for this distance the circuits have an efficiency of 13 miles of standard cable. If unloaded, these circuits would have an efficiency of about 60 miles of standard cable. Over such circuits no commercial service could be given between New York and Philadelphia. Without loading, even 10 gauge conductors would have given unsatisfactory results, so that by means of loading we have been able to obtain results between New York and Philadelphia over

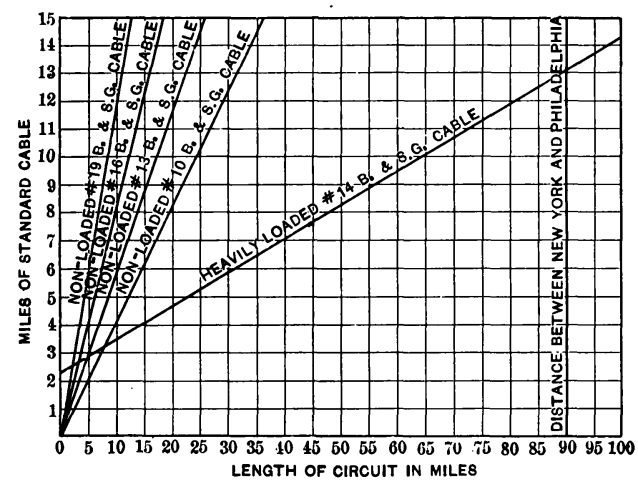

FIG. 6.-Efficiency of No. 14 B. \& S. gauge cable with heavy loading, (New York-Philadelphia cable); terminal losses with terminal transformers and one mile loop; also unloaded cables of various gauges

14 gauge conductors that could not have been obtained with conductors of much greater weight without loading.

While this New York-Philadelphia cable represents the longest loaded underground cable to-day in use anywhere in the world, a project has been approved and is now under way which will far surpass it. This project is for an underground cable ex-' tending between New York and Washington in one direction and New York and Boston in the other. The largest conductors in this cable will be No. 10 B. \& S. gauge and by means of loading we confidently expect that we will be able to obtain thoroughly satisfactory results between New York and either Boston or Washington, and that it will even be possible to obtain service through this cable between Washington and Boston should storm 
damage to the open wire lines render this necessary. The loading of this cable will be of particular interest as it will represent the first large application of the phantoming of loaded cable circuits and the loading of phantom cable circuits. In order to accomplish these results it is necessary to adopt special arrangements of the windings of the loading coils. The windings on the loading coil used for loading the side circuits are arranged as

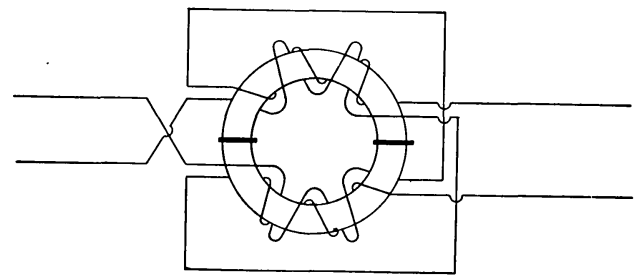

FIG. 7.-Diagram of winding of loading coil for circuits forming the sides of phantom

shown in Fig. 7. With this arrangement of winding, the effect of the telephone current in the side circuit, which current flows in opposite directions in the two sides of the circuit, is to magnetize the core of the loading coil in the same direction throughout and to produce no consequent poles. The loading coil thus acts, in as far as the side circuit is concerned, exactly like the loading coil with the simple winding shown in Figs. 1 and 2 . In building

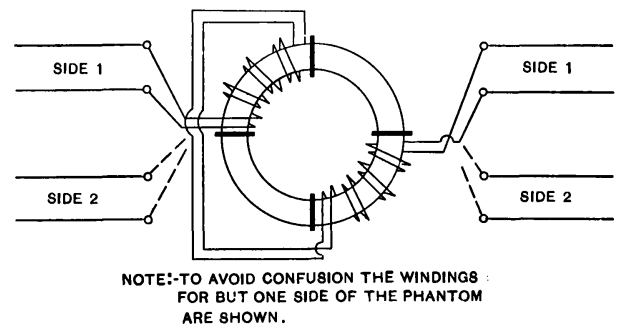

FIG. 8.-Diagram of winding of loading coil for use on phantom circuits

up a phantom circuit this side circuit, however, becomes one side of the phantom, and the telephone current in the phantom circuit traverses the two wires of the side circuit in parallel. Such a current flowing through the windings of such loading coils as are shown in Figs. 1 and 2 would magnetize the two sides of the core in opposite directions and produce consequent poles at the two sides of the core. This would not only bring in 
serious losses, but also crosstalk which would be very difficult to deal with. The current in the phantom circuit flowing through a coil with windings on it as shown in Fig. 7 does not magnetize the loading coil at all, as each winding is neutralized by a corresponding winding on the other side of the line. Such coils may be used to effectively load the side circuits of a phantom combination and in as far as the phantom itself is concerned they act only as resistance which is very small in amount. This, however, does not load the phantom circuit, which of course is a desirable result to attain. To load the phantom circuit a coil with windings as shown in Fig. 8 is employed. It will be seen by tracing out the winding of this coil that to the current flowing in the phantom circuit it acts like an ordinary loading coil, that is, all windings magnetize the core in the same direction. To currents flowing in either of the side circuits, however, it acts only as dead resistance. The arrangements described above in connection with the application of loading coils to phantom circuits and to the side circuits of phantom circuits have been successfully applied to open wire lines as well as to underground cables.

\section{Loading Open Wire Lines}

Gratifying as have been the results obtained from the use of loading on underground cables, its application to open wire circuits has also resulted in most noteworthy improvements in their efficiency. The general plan of loading open wire circuits is similar to that used on underground cable wires. On account of the differences in the electrical characteristics of open wire circuits, as compared with cable circuits, however, the design and spacing of the loading coils is materially different from the coils and the spacings used on underground cables. In general, only heavy loading is used on open wire lines on account of the greater length of these lines. The coils themselves are substantially larger than those employed on cable circuits for two reasons-in the first place the coil is subject to damage by lightning. In order to minimize this difficulty the coils must be built to have high breakdown strengths against lightning discharges. All aerial coils are built so that they will stand a breakdown test of 8,000 volts. This, of course, considerably increases the size of the coil. The coil is further increased in size by the fact that, as an aerial circuit is initially a good deal more efficient than an underground circuit, it is necessary that the coils used in loading it should have smaller losses than are 
permissible in underground cable loading coils. The outside diameter of the aerial loading coil is about 10 inches and the resistance of the two windings of the coil to direct current is $2.5 \mathrm{ohms}$ while to alternating currents of a frequency of 800 periods a second it is $6.5 \mathrm{ohms}$. In addition to building the coil so that it has a breakdown strength of 8,000 volts, each coil is protected by a pair of lightning arresters adjusted to operate at 3,500 volts. These precautions have proved sufficient to reduce trouble from lightning on aerial loading coils to a negligible amount.

Low insulation which is sometimes encountered on open wire lines, particularly in periods of bad weather, proved at the start

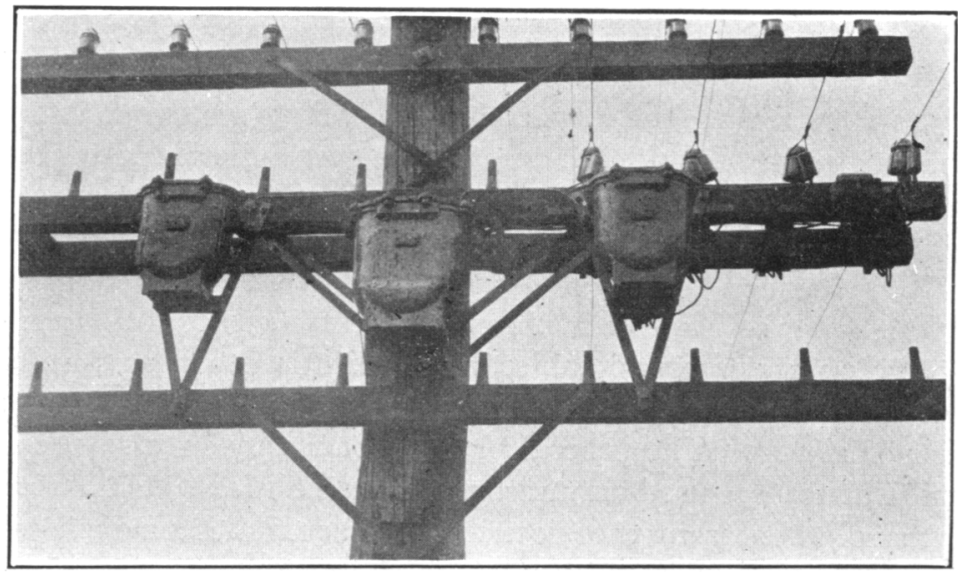

FIG. 9.-Loading coils placed on an open wire line

a serious difficulty in connection with the loading of open wire lines. This, however, has been overcome by removing the worst causes of low insulation and in some cases by the use of improved insulators, and at the present time low insulation is not proving a serious factor in connection with the application of loading to overhead lines. In wet weather the transmission efficiency of overhead lines is not quite as good as in dry weather, but it may be stated that it is, very seldom that the loaded circuits fall in efficiency sufficiently to seriously affect the transmission, except in cases where the lines are in actual trouble from branches of trees falling into the line or other similar causes.

In connection with the loading of open wire lines the problem 
of arranging them so that they will be free from substantial crosstalk has required attention. Due to the fact that much of the crosstalk disturbance in telephony are from electrostatic induction it is to be expected and it is found in practice that the loading of a circuit increases its tendency to crosstalk. In the early applications of loading to open wire lines some trouble was experienced from crosstalk and special transposition systems were devised to overcome these difficulties and it was further found necessary to locate the loading coils with special reference to the transpositions. On underground cables it has been found that loading also increases the crosstalk somewhat, and in these we arrange to reduce the capacity unbalances in order to reduce crosstalk to a sufficiently low point.

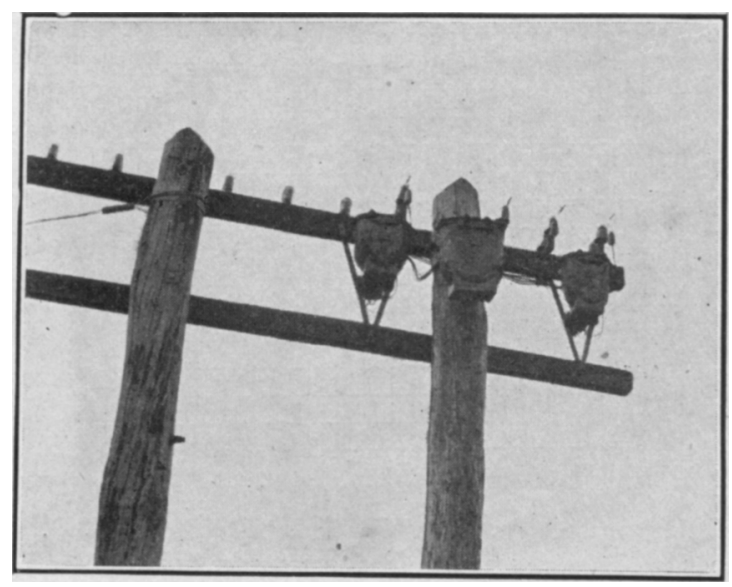

FIG. 10.-Loading coils placed on an open wire line on " $H$ " Fixtures

The loading coils for use on open wire circuits are placed in individual cast iron cases and these are placed on the poles or crossarms as in Figs. 9 and 10 which show illustrations of two common arrangements. The line is carried to the loading coil by means of bridle wire. The lightning arrester is placed in a separate case and bridle wire is also extended to it.

The results obtained by the use of loading coils on the No. 8 B. W. and No. 12 N. B. S. gauge wires, which are the standard sizes used in the long distance plant of the Bell system, are shown in Fig. 11, in which for the purposes of comparison the efficiencies of the unloaded circuits are also given. This diagram 
shows terminal losses with the conditions which may be considered as average. The general reflection loss problem with overhead lines is the same as with underground cables, and having been discussed in detail in connection with such circuits it need not be repeated here. As will be seen from Fig. 11 the results obtained from loading open wire lines have been to more than double their transmission efficiency when used in considerable lengths.

One of the very valuable results which has followed from the loading of open wire lines has been that it has made a very sub. stantial extension to the distance over which long distance service can be given. Up to the time that loading was successfully applied to No. 8 B. W. G. wires, about 1,000 miles repre-

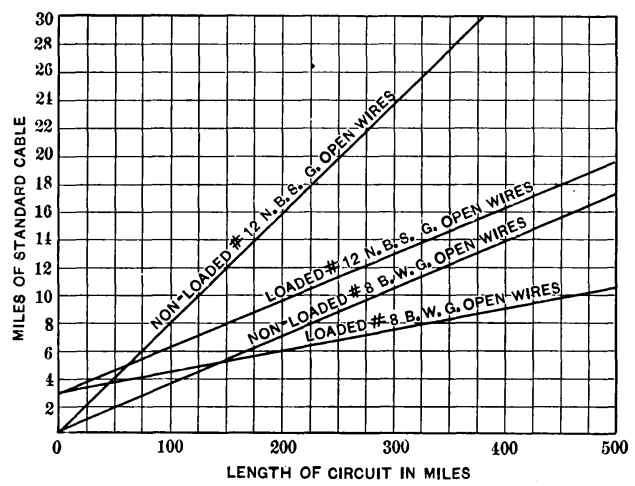

FIG. 11.-Efficiency of No. 8 B. W. G. and No. 12 N. B. S. G. open wire lines loade 1 and unloaded with average terminal con ditions

sented the greatest distances over which commercial service was maintained. By the loading of No. 8 circuits this has just been extended to 2,000 miles, so that to-day it is practicable to obtain a successful telephone conversation from New York to Denver. The actual distance between these two points by the route which the line follows is 2,010 miles.

A very important use has been made of loaded cables in bringing loaded open wire lines_into large cities. Loaded cables have also been used to bring unloaded open wire lines into thickly settled communities, but in this case the gains have not been so great because it is not practicable to place terminal transformers for reducing the reflection losses between the outer end of the underground cable circuits and the aerial lines, and hence reflection losses are experienced. 
This practical impossibility of placing reflection reducing devices between open wire and cable has also made it impossible to obtain the full benefits from loading in those cases where short stretches of cable are placed in the middle of toll linese.g.,. in communities where the appearance of the open wires is objected to and requests are made to place them underground. If the open wires are loaded the trouble is not so great as a comparatively heavy loading can be used without excessive reflection losses. For non-loaded open wires, the loading of the cable if attempted at all must be very light if the reflection losses are not to offset any gain from the reduced attenuation.

For these reasons and also because cable circuits loaded or non-loaded are inherently less efficient than open wire circuits it is important that every effort be made to keep toll lines as free as possible from cable.

\section{Loading of Submarine Cables}

The problem of loading submarine cables is a mechanical one rather than one concerning the principles of loading. In this country a 16 B. \& S. G. dry core paper submarine cable about 5 miles long and containing loading coils spaced about 2 miles apart has been successfully laid across the Chesapeake Bay and is now in operation. There has also been talk of laying a dry core loaded submarine cable across Puget Sound for a distance of 16 miles. The situation in the United States, however, is not such that large numbers of loaded submarine cables of any great length are required. Abroad, however, particularly between England and the continent and between England and Ireland submarine cables of considerable length are needed for telephone purposes and in these situations loaded gutta-percha submarine cables have been employed with successful results.

\section{Extent of Application of Loading}

It is evident that a device making such great improvements in the transmission efficiency of telephone circuits as does loading would have a large place in the plant of any company providing a comprehensive system of telephone service over a large area, a condition necessarily requiring long cables and long open wire lines. Although the loading art was born in 1900, and its successful commercial application on an extensive scale to our plant dates only from about 1903, there are already in the United States over 85,000 miles of loaded open wire circuits and over 
170,000 miles of loaded underground cable circuits. To load these circuits there have been employed about 125,000 loading coils. The day-by-day experience with this enormous loaded line plant has fully demonstrated that we are in practice obtaining the successful results which the engineering data that I have set forth would lead us to expect.

The facts which have been herewith developed have, I think, been more than sufficient to justify the statement at the beginning of this paper, that the year 1900, in which the loading patents were issued to Professor Pupin, marked the beginning of an important era in telephone work. I do not know of any problem in electrical science and electrical engineering which has required greater skill, technical ability and diligence on the part of those engaged in its solution-inventors, engineers, manufacturers and construction people-than has the working out and the application of loading, and it is a pleasure to be able to present to you a statement of the results which they have accomplished. 